\title{
Stress Hyperglycemia and Complications Following Traumatic Injuries in Individuals With/Without Diabetes: The Case of Orthopedic Surgery
}

This article was published in the following Dove Press journal: Diabetes, Metabolic Syndrome and Obesity: Targets and Therapy

\author{
Raffaella Di Luzio' \\ Rachele Dusi ${ }^{2}$ \\ Arianna Mazzotti ${ }^{2,3}$ \\ Maria Letizia Petroni ${ }^{2}$ \\ Giulio Marchesini (iD ${ }^{2}$ \\ Giampaolo Bianchi $\mathbb{I D}^{2}$ \\ 'Unit of Internal Medicine - AUSL \\ Bologna, Bologna, Italy; ${ }^{2}$ Department of \\ Medical and Surgical Sciences (DIMEC), \\ "Alma Mater" University, Bologna, Italy; \\ ${ }^{3}$ Diabetes Unit, AUSL Romagna, Ravenna, \\ Italy
}

Purpose: Hyperglycemia in trauma patients may stem from metabolic response to stress, both in the presence and the absence of underlying diabetes. We aimed to test the association of stress hyperglycemia with risks of adverse events subjects undergoing orthopedic surgery. Patients and Methods: In a prospective observational study, we enrolled 202 consecutive patients with hyperglycemia at hospital admission for trauma injuries requiring orthopedic surgery. Based on history, diabetes was present in 183, and 13 more were defined as unknown diabetes on the basis of $\mathrm{HbA} 1 \mathrm{c} \geq 48 \mathrm{mmol} / \mathrm{mol}$. Stress hyperglycemia was defined in subjects with/without diabetes by a stress hyperglycemia ratio (SHR) $>1.14$, calculated as admission glucose/average glucose, estimated from glycosylated hemoglobin. Logistic regression analysis was used to calculate the risk of post-surgery adverse events associated with different states of hyperglycemia, after correction for demographic and clinical confounders.

Results: Stress hyperglycemia was diagnosed, either as superimposed to diabetes $(54 / 196$ cases, $27.6 \%$ ) as well as in the 6 cases without diabetes. At least one complication was recorded in 68 cases $(33.7 \%)$, the most common being systemic infection (22.8\% of cases). In the total cohort, stress hyperglycemia, irrespective of the presence of diabetes, increased the risk of adverse events (any events, odds ratio [OR], 4.43; 95\% confidence interval [CI], 2.11-9.30), cardiovascular events (OR, 7.09; 95\% CI, 2.47-19.91), systemic infections (OR, $4.21 ; 95 \%$ CI, 1.97-9.03) and other adverse events (OR, 6.30; 95\% CI, 1.41-28.03), after adjustment for confounders; hospital stay was much longer. The same was true when the analysis was limited to the diabetes cohort or by comparing pure stress hyperglycemia vs diabetes without stress hyperglycemia.

Conclusion: The study highlights the importance of stress hyperglycemia for adverse events in the setting of orthopedic surgery following trauma injuries. This condition requires stricter management, considering the much longer length of hospital stay and higher costs.

Keywords: adverse events, comorbidities, hospital stay, infections, trauma

\section{Introduction}

Hyperglycemia is common in daily practice with hospitalized individuals, present in nearly $25-35 \%$ of Italian patients at admission. ${ }^{1}$ The prevalence is dictated by the high prevalence of diabetes in the elderly ${ }^{2}$ (much more commonly admitted than young individuals) and by stress-induced hyperglycemia, i.e., the elevation in blood glucose levels during periods of illness, resulting in remarkable metabolic stress. ${ }^{3}$ Mild-to-moderate stress hyperglycemia is protective, providing fuel for the immune system and brain at a time of stress; however, persistent hyperglycemia and insulin
Correspondence: Giulio Marchesin Department of Medical and Surgical Sciences (DIMEC), "Alma Mater"

University, Sant'Orsola-Malpighi Hospital, Bologna I-40I38, Italy

Tel +390512144889

Fax +3905I 6364502

Email giulio.marchesini@unibo.it 
resistance may be potentially deleterious in the long run, ${ }^{4}$ directly contributing to adverse outcomes via endothelial dysfunction, increased free radical production (oxidative stress), inflammatory responses, vascular and immune dysfunction. ${ }^{5}$

A relationship between admission plasma glucose concentration and in-hospital mortality has long been established. Hyperglycemia has been associated with increased risks of congestive heart failure and mortality, both during the acute phase and the long-term follow-up of myocardial infarction, ${ }^{4}$ of mortality for community-acquired bacteremia, ${ }^{6}$ of acute respiratory failure in subjects with chronic obstructive pulmonary disease, ${ }^{7}$ as well as of mortality, poor functional recovery and transformation into hemorrhagic stroke in subjects with ischemic vascular disease. ${ }^{8,9}$

Acute hyperglycemia following traumatic injury has also received a lot of attention; traumatic injuries induce stress hormone secretion, largely mediated by glucagon, ${ }^{10}$ in turn promoting oxidative stress and insulin resistance, finally resulting in hyperglycemia. ${ }^{11-14}$ Admission blood glucose represents a clinically useful predictor of mortality and infectious outcome in traumatically injured patients, ${ }^{15,16}$ and achieving normoglycemia in the early post-traumatic period may reduce the risk. ${ }^{17}$

The definition of stress hyperglycemia remains difficult at the time of the event. Without evidence of prior diabetes, hyperglycemia during acute events could also stem from undiagnosed diabetes, where the elevated blood glucose remains an independent risk factor for complication. A recent study defined relative hyperglycemia (stress hyperglycemia ratio $[\mathrm{SHR}]$, ie, admission glucose divided by the estimated average glucose derived from glycosylated hemoglobin) as a tool to detect stress hyperglycemia. ${ }^{18}$ As such, SHR controls for background blood glucose and was shown to be a better biomarker of critical illness than absolute hyperglycemia. ${ }^{18}$

We tested the reason(s) for hyperglycemia in a consecutive series of subjects undergoing orthopedic surgery in order to determine the presence of stress hyperglycemia and its association with the final outcome. Data may be relevant for tailored intervention and proper management.

\section{Materials and Methods}

\section{Patients}

This was an observational, prospective cohort study carried out in two Orthopedics units of the University Hospital Sant'Orsola-Malpighi and Rizzoli Orthopedic Institute of Bologna (Italy). The study included consecutive patients aged $\geq 18$ years, admitted with injuries requiring acute intervention and hyperglycemia at the time of admission. Patients with orthopedic injuries non-requiring operative intervention, under corticosteroid treatment or critical illnesses were excluded.

The demographic and clinical data at entry are reported in Table 1. The distinction between minor or major surgery was obtained by the use of current tables of anesthesia and resuscitation Units (Supplementary Material). Following the demonstration of hyperglycemia, all cases were insulin-treated until glucose normalization or discharge from hospital, when they were referred to specialized diabetes units. The whole study received approval by the ethical committees of the participating institutions and was conducted according to the Helsinki declaration. The patients signed an informed consent to participation and anonymous data collection.

\section{Methods}

Admission glucose was recorded as the first glucose measurement after admission; hyperglycemia was defined as: a) fasting glucose values $\geq 126 \mathrm{mg} / \mathrm{dL}$ ( $7 \mathrm{mmol} / \mathrm{L}$ ) from venous blood or capillary blood; b) capillary or venous blood glucose levels $\geq 144 \mathrm{mg} / \mathrm{dL}$ ( $8 \mathrm{mmol} / \mathrm{L}$ ) in patients tested in non-fasting conditions.

Patient information was recorded and included demographic characteristics, medical history, blood glucose on admission and during hospital stay, HbAlc, concurrent medical diagnoses, diet and/or drug treatment for glucose control, length of stay, and hospital outcome. From patient history, we calculated the age-adjusted Charlson Comorbidity Index. ${ }^{19}$ During the hospital stay, insulin treatment was immediately instituted and daily blood glucose was monitored with four bed-side capillary glucose measurements (three pre-meal blood glucose measurements and 1-2 hours after the evening meal) in pre- or post-surgical days or more frequently during the day of surgery.

Total hospital stay, needs for admission to intensive care unit (ICU) and re-intervention were recorded, as well as adverse events, with special consideration for local and systemic infections and cardiovascular (CV) events. Perioperative infectious complications were categorized as pneumonia, urinary tract infections, and surgical site infections. Surgical-site infection was defined as any case that required reoperation for an infection at the site of the index operation and was confirmed by the presence of either positive intraoperative cultures, pathology specimens with microbiologic pathogens, or visible gross purulence at the operative site. 
Table I Baseline Demographic and Clinical Data from Patients of the Total Cohort. The Age-Adjusted Charlson Comorbidity Index Was Calculated on the Basis of Medical History ${ }^{19}$

\begin{tabular}{|c|c|}
\hline Clinical Characteristics & $\begin{array}{l}\text { All Patients } \\
(n=202)\end{array}$ \\
\hline Age (years) & $74.4 \pm 12.4$ \\
\hline Weight (kg) & $76.4 \pm 15.2$ \\
\hline Height $(\mathrm{cm})$ & $165.1 \pm 9.8$ \\
\hline Body Mass Index $\left(\mathrm{kg} / \mathrm{m}^{2}\right)$ & $27.9 \pm 4.5$ \\
\hline Glycosylated Alc hemoglobin (\%) & $7.08 \pm 1.00$ \\
\hline Blood glucose (mg/dL) & $164.7 \pm 59.8$ \\
\hline Serum hemoglobin $(g / d L)$ & $12.6 \pm 1.8$ \\
\hline Serum potassium (mEq/L) & $4.43 \pm 0.54$ \\
\hline Serum creatinine $(\mathrm{mg} / \mathrm{dL})$ & $1.00 \pm 0.47$ \\
\hline Age-adj Charlson Comorbidity Index & $5.21 \pm 2.12$ \\
\hline Major surgery (\%)* & $85.6(79.9-89.6)$ \\
\hline \multicolumn{2}{|l|}{ Comorbidities (\%) } \\
\hline Arterial hypertension (\%) & $76.7(70.2-81.8)$ \\
\hline Dyslipidemia (\%) & $37.1(30.5-43.7)$ \\
\hline Coronary artery disease (\%) & $17.3(12.5-22.9)$ \\
\hline Peripheral vascular disease (\%) & $\mid 5.8(|| .2-2 \mid .2)$ \\
\hline Chronic kidney disease (\%) & $10.9(7.1-15.7)$ \\
\hline Retinopathy (\%) & $6.4(3.6-10.4)$ \\
\hline Previous cancer (last 5 years)(\%) & $12.9(8.7-17.9)$ \\
\hline Liver disease (\%) & $9.4(5.9-13.9)$ \\
\hline Previously diagnosed diabetes $(\mathrm{N}=183)(\%)^{*}$ & $90.6(85.5-93.7)$ \\
\hline Newly-detected diabetes $(\mathrm{N}=\mid 3)(\%)$ & $6.4(2.8-13.2)$ \\
\hline \multicolumn{2}{|l|}{ Diabetes treatment } \\
\hline Diet only $(\mathrm{N}=\mid 8)(\%)^{\circ}$ & $9.8(6.1-14.6)$ \\
\hline Metformin $(\mathrm{N}=63)(\%)^{\circ}$ & $34.4(27.7-4 \mid .3)$ \\
\hline Any additional non-insulin drug $(\mathrm{N}=55)(\%)^{\circ}$ & $29.9(23.5-36.6)$ \\
\hline Insulin + any non-insulin treatment $(\mathrm{N}=21)(\%)^{\circ}$ & II.4 (7.4-I6.5) \\
\hline Insulin alone $(\mathrm{N}=26)(\%)^{\circ}$ & I4.I (9.8-19.6) \\
\hline Relative hyperglycemia (SHR) & $1.06 \pm 0.34$ \\
\hline Stress hyperglycemia $(\mathrm{SHR}>\mathrm{I} .14)(\mathrm{N}=58)(\%)^{*}$ & $28.9(22.8-35.2)$ \\
\hline
\end{tabular}

Notes: Data are presented as means \pm standard deviation. *Percent $(95 \%$ confidence interval). ${ }^{\#}$ Estimated glomerular filtration rate $<60 \mathrm{~mL} / \mathrm{min} / 1.73 \mathrm{~m}^{2}$ (with/ without albuminuria). ${ }^{\circ}$ Percent within diabetes cohort ( $95 \%$ confidence interval).

\section{Laboratory Tests}

$\mathrm{HbAlc}$ is measured in the whole area of Bologna by a single laboratory, certified according to the initial DCCT rules and later as from the National Glycohemoglobin Standardization Program (NGSP). HbAlc was used to detect an unknown diabetic state $(\mathrm{HbA} 1 \mathrm{c} \geq 48 \mathrm{mmol} / \mathrm{mol}),{ }^{1}$ as well as to estimate the average blood glucose concentration before admission using the equation: estimated average glucose $(\mathrm{mg} / \mathrm{dL})=$ $(28.7 * \mathrm{HbAl} 1 \mathrm{c}[\%])-46.7 .^{20}$

Relative hyperglycemia was defined by SHR, calculated as admission glucose divided by estimated average glucose. ${ }^{18}$ Values of SHR $>1.14$ were considered as indicative of stress-induced hyperglycemia, both in the presence and the absence of previously known diabetes. Using diabetes history, HbAlc and SHR as indicators, we grouped patients into three categories (Figure 1): a) diabetes/no stress hyperglycemia (SHR $\leq 1.1 .14)$; b) diabetes/stress hyperglycemia (SHR $>1.14$ ): c) no diabetes/ stress hyperglycemia (SHR $>1.14$ ).

We also categorized patients with stress hyperglycemia into quartiles of SHR in order to test the possible incremental role of stress hyperglycemia on the increased risk of complications.

\section{Statistical Analysis}

Statistical analysis was performed using StatView 5.0 (SAS Institute Inc., Cary, NC) and SPSS for Windows v.21 (SPSS Inc., Chicago, IL, USA). Continuous variables were reported using mean and standard deviation (SD) or median and interquartile range (IQR), as appropriate. Absolute prevalence and percentage were used for categorical variables (dichotomous variables). Differences between continuous variables for the different cohorts were tested for significance using the MannWhitney $U$-test. Prevalence in different groups/subgroups was compared with Chi-squared test or with Fisher's exact test when appropriate.

A logistic regression analysis was performed to ascertain the association of stress hyperglycemia with the different outcome variables, after adjusting for age, sex, body weight (BMI), comorbidities (age-adj Charlson's index) and type of surgery. The relative risk of outcome variables was calculated as odds ratios (OR) and $95 \%$ confidence interval (CI). $\mathrm{P}$ values $<0.05$ were considered statistically significant.

\section{Results}

\section{Admission Values}

Two hundred and two patients satisfied the inclusion criteria and were considered in the analysis. Their baseline demographic data are reported in Table 1. The average body mass index (BMI) was $28.0 \mathrm{~kg} / \mathrm{m}^{2}$, in a wide range 


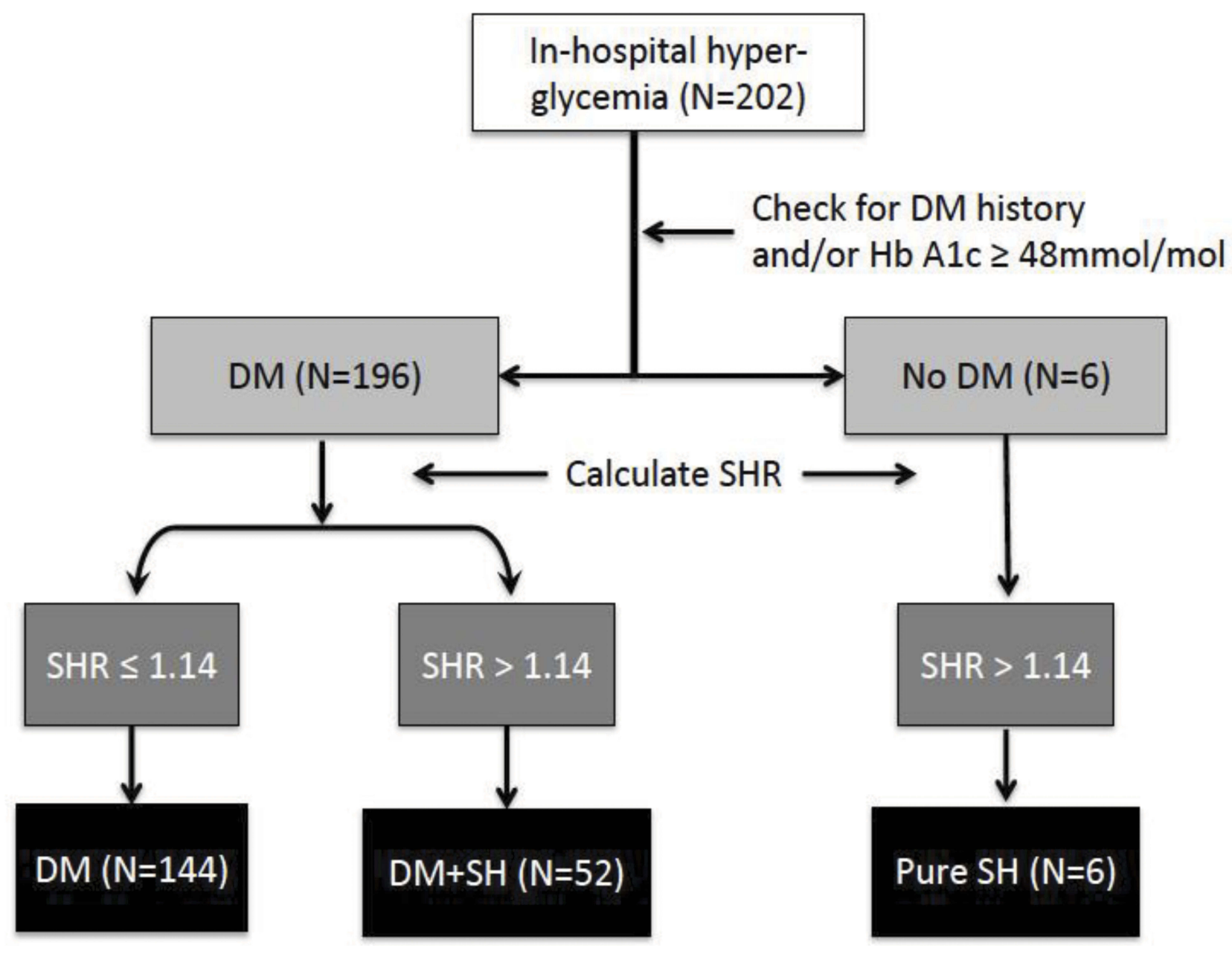

Figure I Diagnostic algorithm for the evaluation of patients with in-hospital hyperglycemia at admission (number of cases are reported in parenthesis). Abbreviations: DM, diabetes mellitus; HbAlc, glycosylated hemoglobin; SH, stress hyperglycemia (SHR >I.I4); SHR, stress hyperglycemia ratio.

(from 15.6 to 44.1). One hundred and seventy-eight patients had a history of type 2 diabetes, 5 had Type 1 diabetes, and 19 showed hyperglycemia at hospital admission in the absence of a previous history of diabetes. In 13 cases, $\mathrm{Hb}$ Alc $\geq 48 \mathrm{mmol} / \mathrm{mol}$ revealed a previously unreported diabetes. Most patients with diabetes were being treated with oral glucose-lowering agents alone, 23\% with insulin (alone or in combination with oral agents), and the remaining $18 \%$ with the sole diet. In most cases $(n=173$, $85.6 \%$ ), surgical treatment fell within the criteria of major surgery. The average time from admission to surgery was 2.3 days (2.45 days in patients undergoing major surgery, 1.89 days for minor surgery), while 8.7 days was the average time from surgery to hospital discharge (9.58 days for major surgery; 4.58 days for minor surgery).

Several associated diseases were also recorded, as were diabetes-related comorbidities ( $16.3 \%$ of total cases). The age-adjusted Charlson comorbidity index was on average
$5.2 \pm 2.1$, median 5.0 points; the score was neither different between genders nor between subjects in the three different cohorts.

\section{Glucose Values and Categories of Hyperglycemia}

The number of cases fitting the different classes of hyperglycemia are reported in Figure 1.

In 53/196 cases with diabetes (26.5\%), hyperglycemia was exaggerated in comparison to the average blood glucose estimated from glycosylated hemoglobin (SHR >1.14) and the patients were considered to have stress hyperglycemia superimposed to diabetes. SHR was $\geq 1.14$ also in the remaining 6 cases, considered to represent pure stress hyperglycemia.

In comparison to subjects with diabetes and no stress hyperglycemia (reference) (Table 2), the presence of stress hyperglycemia superimposed to diabetes was characterized 
Table 2 Principal Characteristics and Adverse Events or Complications in Relation to the Presence of Diabetes and Stress Hyperglycemia

\begin{tabular}{|c|c|c|c|c|c|}
\hline Variable & $\begin{array}{l}\text { Diabetes, No SH } \\
(\mathrm{N}=143)\end{array}$ & $\begin{array}{l}\text { Diabetes, } \mathrm{SH+} \\
(\mathrm{N}=53)\end{array}$ & $\begin{array}{l}\text { No Diabetes, } \mathrm{SH}+ \\
(\mathrm{N}=6)\end{array}$ & $\begin{array}{l}\text { P value } \\
\text { DM (No SH } \\
\text { vs. SH+) }\end{array}$ & $\begin{array}{l}\text { P value } \\
\text { SH+ vs No } \\
\text { SH }\end{array}$ \\
\hline Admission glucose $(\mathrm{mg} / \mathrm{dL})$ & $140.6 \pm 34.5$ & $230.1 \pm 68.0$ & $159.8 \pm 7.8$ & $<0.001$ & $<0.001$ \\
\hline $\mathrm{HbAlc}(\mathrm{mmol} / \mathrm{mol})$ & $7.11 \pm 1.01$ & $7.09 \pm 0.97$ & $6.10 \pm 0.38^{\wedge}$ & 0.887 & 0.425 \\
\hline Relative hyperglycemia & $0.90 \pm 0.17$ & $1.47 \pm 0.37$ & $1.26 \pm 0.13$ & $<0.001$ & $<0.001$ \\
\hline Age (years) & $73.5 \pm 12.7$ & $76.0 \pm 11.6$ & $83.3 \pm 7.7$ & 0.136 & 0.085 \\
\hline Age-adj Charlson Index & $5.08 \pm 2.17$ & $5.55 \pm 2.01$ & $5.50 \pm 1.76$ & 0.171 & 0.156 \\
\hline Type of surgery (Major) & $120(83.9,76.6-88.7)$ & $47(88.7,76.3-94.1)$ & $6(100.0,51.7-100.0)$ & 0.500 & 0.378 \\
\hline Cases with events & $33(23.1,16.6-30.3)$ & $29(54.7,40.6-66.5)$ & $5(83.3,36.6-94.0)$ & $<0.001$ & $<0.001$ \\
\hline Cardiovascular events & $6(4.2,1.7-8.4)$ & $10(18.9,9.9-30.3)$ & $4(66.7,24.1-86.1$ & $<0.001$ & $<0.001$ \\
\hline AMl/angina & $2(1.4,0.2-4.4)$ & $2(3.8,0.7-11.4)$ & I $(16.7,0.9-48.3)$ & 0.296 & 0.150 \\
\hline Stroke/TIA & $0(0.0-1.9)$ & I $(1.9,0.1-8.4)$ & $0(0.0-27.3)$ & 0.270 & 0.292 \\
\hline Heart failure & I $(0.7,0.0-3.3)$ & $5(9.4,3.5-19.1)$ & I $(16.7,0.9-48.3)$ & 0.006 & 0.003 \\
\hline Arrhythmias & $4(2.5,0.4-7.7)$ & $5(9.4,3.5-19.1)$ & $2(33.3,6.0-63.5)$ & 0.062 & 0.016 \\
\hline Local infections & $3(2.1,0.5-5.5)$ & $2(3.8,0.7-11.4)$ & $0(0.0-27.3)$ & 0.613 & 0.630 \\
\hline Systemic infections & $21(14.7,9.5-21.0)$ & $22(4 I .5,28.4-54.0)$ & $3(50.0,13.9-75.9)$ & $<0.001$ & $<0.001$ \\
\hline Other complications & $3(2.1,0.5-5.5)$ & $6(|1.3,4.7-2| .4)$ & I $(16.7,0.9-48.3)$ & 0.013 & 0.007 \\
\hline ICU admission & $6(4.2,1.7-8.4)$ & $4(7.5,2.4-16.6)$ & I $(16.7,0.9-48.3)$ & 0.464 & 0.304 \\
\hline Reintervention & $0(0.0-1.9)$ & $3(5.7,1.5-14.1)$ & I $(16.7,0.9-48.3)$ & 0.117 & 0.007 \\
\hline
\end{tabular}

Notes: Data are presented as means \pm SD or as number of cases (\%, $95 \%$ confidence interval). ^Student $t$-test, Chi ${ }^{2}$ test or Fisher exact test, as appropriate). Abbreviations: AMI, acute myocardial infarction; ICU, intensive care unit; TIA, transient ischemic attack; SH, stress hyperglycemia.

by higher blood glucose levels at admission, but no systematic differences in $\mathrm{HbAlc}$, age and comorbidities.

\section{In-Hospital Events}

During hospitalization, patients were monitored for the development of post-operative complications, including the possible need for a re-intervention, unplanned admissions to ICU and death. At least one complication was recorded in 68 cases $(33.7 \%)(32.2 \%$ following major surgery, $1.5 \%$ after minor surgery). The most common complication was systemic infection (22.8\% of cases), with/without local infection at the surgical site, which occurred alone in $7.3 \%$ of cases.

As to cardiovascular complications, $2.5 \%$ of patients developed a coronary event, $3.5 \%$ acute heart failure and $5 \%$ atrial fibrillation. Only one patient experienced a transient cerebrovascular accident $(0.5 \%)$, while no patients developed acute renal failure. Another $5 \%$ of cases were complicated by a miscellanea of obstructive airway episodes, acute cholecystitis, respiratory failure, epileptic crises, pericarditis, partial bowel obstruction and acute liver failure; $2.5 \%$ of cases experienced at least one hypoglycemic episode. Unplanned admission to ICU was required in 5.4\% of patients, while $4(2 \%)$ required re-intervention. Only one patient $(0.5 \%)$ died during hospitalization; $43.6 \%$ received at least one unit of packed red blood cells.
The rates of complicating events were different in relation to categories of hyperglycemia. In comparison to subjects with diabetes and no stress hyperglycemia (reference) (Table 2), the presence of stress hyperglycemia superimposed to diabetes was characterized by higher rates of cardiovascular events and systemic infections. These complications were very common also in the small group of cases with stress hyperglycemia in the absence of diabetes. When all cases with stress hyperglycemia were grouped, the resulting cohort was also characterized by significantly higher rates of arrhythmias and reintervention (Table 2).

\section{Outcomes}

The association of hyperglycemia category with outcomes is reported in Table 3, where diabetes without stress hyperglycemia was considered as the reference category. In the whole population with diabetes, stress hyperglycemia significantly increased the risk of adverse events, excluding local infections. In subjects with stress hyperglycemia without diabetes, only the significant association with cardiovascular events was maintained, with the limits of a limited number of cases. When all cases with stress hyperglycemia were merged, stress hyperglycemia was associated with four-time increased risk of adverse events, over ten-time increased risk of cardiovascular events, 
Table 3 Association of Hyperglycemia Categories with Adverse Events in Subjects with/Without a History of Diabetes

\begin{tabular}{|c|c|c|c|c|c|c|}
\hline \multirow[t]{2}{*}{ Hyperglycemia Categories } & \multirow{2}{*}{$\begin{array}{l}\text { All Adverse } \\
\text { Events }(\mathrm{N}=68)\end{array}$} & \multicolumn{5}{|c|}{ Odds Ratio ( $95 \%$ Confidence Intervals) } \\
\hline & & $\begin{array}{l}\text { CV Events* } \\
(\mathrm{N}=19)\end{array}$ & $\begin{array}{l}\text { Systemic } \\
\text { Infections ( } \mathrm{N}=46)\end{array}$ & $\begin{array}{l}\text { Local } \\
\text { Infections } \\
(\mathrm{N}=5)\end{array}$ & $\begin{array}{l}\text { Admission to } \\
\text { ICU }(\mathrm{N}=10)\end{array}$ & $\begin{array}{l}\text { Other Events }^{\circ} \\
(\mathrm{N}=10)\end{array}$ \\
\hline $\begin{array}{l}\text { I. Diabetes, no SH (reference) } \\
(\mathrm{n}=154)\end{array}$ & - & - & - & - & - & - \\
\hline 2. Diabetes, $S H+(n=42)$ & $4.03(1.95-8.32)$ & $\begin{array}{l}5.51 \\
(1.83-18.80)\end{array}$ & $4.32(1.94-9.62)$ & $2.19(0.55-8.68)$ & $1.95(0.44-8.66)$ & $\begin{array}{l}6.62 \\
(1.37-31.95)\end{array}$ \\
\hline 3. No diabetes, $\mathrm{SH}+(n=6)$ & $13.08(1.25-100.3)$ & $\begin{array}{l}44.52 \\
(4.70-422.1)\end{array}$ & $3.78(0.63-22.63)$ & na & $4.13(0.34-50.01)$ & $\begin{array}{l}11.18 \\
(0.50-249.3)\end{array}$ \\
\hline $\begin{array}{l}\text { 8. Stress hyperglycemia (all cases: } \\
2+3)(n=48)\end{array}$ & $4.43(2.11-9.30)$ & $\begin{array}{l}7.09 \\
(2.47-19.91)\end{array}$ & $4.21(1.97-9.03)$ & $3.40(0.46-24.95)$ & $2.37(0.65-8.55)$ & $\begin{array}{l}6.30 \\
(1.41-28.03)\end{array}$ \\
\hline
\end{tabular}

Notes: All data are adjusted for age, sex, body mass index, Charlson's index and type of surgery. Statistically significant results are presented in bold characters. $* \mathrm{CV}$ complications include acute myocardial infarction, arrhythmias, left bundle branch block, transient ischemic cerebral attack, stroke. ${ }^{\circ}$ Other events include pulmonary, neurological and hepatic complications.

Abbreviations: $\mathrm{SH}$, Stress hyperglycemia; CV, cardiovascular; ICU, intensive care unit; na, data not calculated because of lack of events.

four-time risk of systemic infections, six-time increase of other adverse events, without changes in the risk of ICU admission, after adjustment for confounders. In a sensitivity analysis, SHR as continuous variable was associated with an increased risk of adverse events (any events) in the whole population (adjusted Odds ratio [adj OR], 1.25 per 0.10 increment; $95 \%$ confidence interval [CI], $1.12-1.39 ; \mathrm{P}<0.0001$ ), irrespective of the presence of diabetes.

After exclusion of the five cases with type 1 diabetes, the association of stress hyperglycemia with complications (any events) (adj OR 4.11, 95\% CI 1.98-8.51), with systemic infection (adj OR 4.40, 95\% CI 2.06-9.40) and with cardiovascular events (adj OR 6.88, 95\% CI 2.42-19.57) was maintained. The same was also true after exclusion of all insulin-treated subjects $(\mathrm{n}=26)$ (any events, adj $\mathrm{OR}$ 3.93, 95\% CI 1.62-9.54; systemic infection, adj OR 3.93; 95\% CI 1.62-9.54; cardiovascular events, adj OR 8.93; 95\% CI 2.70-29.49).

The length of hospital stay was much longer in the presence of stress hyperglycemia $(+24.5 \% ; 13.3 \pm 6.2$ days vs $10.7 \pm 7.9$ in subjects without stress hyperglycemia; $\mathrm{P}=$ 0.042).

\section{Discussion}

The study highlights the importance of stress hyperglycemia in the development of complications in the specific setting of subjects undergoing orthopedic surgery following traumatic injuries. Irrespective of the presence of overt or newly-discovered diabetes, the risk of adverse events increases several times whenever blood glucose exceeds the values corresponding to the measured glycosylated hemoglobin. This condition also requires a different management, considering the longer length of hospital stay and is expected to carry much higher costs.

Our analysis is based on the assessment of SHR, a tool to detect stress hyperglycemia suggested and validated in a large clinical setting by Roberts et al. ${ }^{18}$ This tool appears to be particularly useful since it provides an assessment of stressinduced hyperglycemia also in the presence of underlying diabetes, which might blur the response to stress. In the original proposal, SHR was demonstrated to be independently associated with critical illness to a much larger extent than absolute hyperglycemia, largely driven by diabetes and glucoselowering treatment. ${ }^{18}$ The SHR cut-off to define stress hyperglycemia has never been definitely validated. We choose a value of 1.14, i.e., the average value previously shown to be associated with an increased rate of adverse events. ${ }^{18}$ The SHR value also demonstrates a linear effect on the occurrence of adverse events, along the whole range of SHR values. In a post hoc analysis of our population, where subjects with SHR $>1.14$ were divided into quartiles, the odds ratios of adverse events progressively increased from 3.82 (2nd quartile) to 4.16 (3rd quartile) and finally to 6.95 (4th quartile). Similar results were also observed in the original study. ${ }^{18}$ Our results confirm the detrimental effect of this definition of stress hyperglycemia, to be confidently used in future prospective studies. A rapid assessment of stress hyperglycemia might be particularly useful in conditions where surgery should be carried out without 
any delay, as in traumatic injuries, and particularly in older patients, where diabetes may frequently occur.

Dungan et al suggested that stress hyperglycemia might be a physiological response to stress-induced insulin resistance, via a possible modulation of glucose transporters, independently of diabetes. ${ }^{3}$ However, hyperglycemia per se amplifies the inflammatory response, possibly increasing the risk of adverse events. In our setting the risks for adverse events in subjects with stress hyperglycemia in the absence of diabetes are generally elevated compared to diabetes without stress hyperglycemia, although difficult to define due to the limited sample size. In general, the higher risk of adverse events is confirmed to involve different organs and systems, suggesting that a general abnormal response to stress might be the common driver.

If stress hyperglycemia drives complications, the type and target organ is likely to vary according to a specific setting. In surgical patients, the risk for surgical site infection is particularly critical and adds to the cardiovascular risks previously demonstrated in other settings. Several large studies have consistently associated the presence of diabetes with surgical site infections. ${ }^{21-23}$ In a large database on 13,272 patients who underwent primary joint arthroplasty between 2001 and 2011, Chrastil et al found was no increased risk of infection associated with elevated glycosylated hemoglobin, whereas preoperative hyperglycemia was associated with an increased incidence of perioperative joint infection again in keeping of a major risk of stress-induced hyperglycemia. ${ }^{21}$ In a review article and meta-analysis of surgical procedures, Martin et al found an increased risk for surgical site infection associated with diabetes (OR, 1.53; 95\% CI, 1.11-2.12), and lower values for orthopedic surgery (arthroplasty, OR 1, 26), but they also concluded for a higher risk associated with glucose levels, after controlling for the presence of diabetes. ${ }^{22}$

The risk of adverse events associated with high SHR was particularly elevated in subjects without diabetes, who did not receive any treatment for their hyperglycemia. This points to a possible modulatory effect of glucose-lowering treatment on hyperglycemia and opens the question of systematic assessment of blood glucose and immediate and effective treatment with insulin at admission, as suggested by guidelines, independently of the presence of diabetes. Surgical site infections, independently of the presence of diabetes, are universally recognized as quality-of-care indicators. ${ }^{24}$ In an updated review based on an old report from the National Institute for Health and Clinical Excellence, Bock et al concluded that data did not support routine preoperative testing for blood glucose or HbA1c in apparently healthy adult patients undergoing elective noncardiac surgery. However, also in their analysis orthopedic surgery was worth a screening. ${ }^{25}$ The old age of the majority of patients undergoing elective surgery and the multiple comorbidities associated with diabetes are the likely reason for the difference in comparison to other elective surgeries. In our setting insulin treatment was immediately instituted in the presence of hyperglycemia at admission according to predefined protocols, but adverse events were nonetheless very common and clustered in subjects with stress-induced hyperglycemia. Tailored glucose-controlling, insulin-administrating programs are needed to keep infection and non-infection complications to a minimum. ${ }^{26}$ Only a few well-designed controlled studies are available in the orthopedic setting, ${ }^{27}$ and the implementation of effective procedures in frail hyperglycemic patients by dedicated teams requires special attention. ${ }^{28}$

\section{Conclusion}

Stress-induced hyperglycemia remains difficult to define at time of the event. In the setting of orthopedic surgery, our study confirms that the calculation of the stress hyperglycemia ratio provides a measure with a definite clinical significance. Larger studies are needed to define the precise cut-off of the ratio, both in the presence and the absence of overt diabetes, associated with clinical events, considering the prognostic information it provides. It should also be relevant to define how much the persistence of stress hyperglycemia will impact on adverse events.

\section{Abbreviations}

AMI, acute myocardial infarction; BMI, body mass index; $\mathrm{CV}$, cardiovascular; DM, diabetes mellitus; HbA1c, glycosylated hemoglobin; ICU, intensive care unit; $\mathrm{SH}$, stress hyperglycemia; SHR, stress hyperglycemia ratio; TIA, transient ischemic attack.

\section{Acknowledgments}

The authors are indebted to Prof. Massimo Laus (Head, Orthopedic Unit, Sant'Orsola-Malpighi Hospital) and to Prof. Riccardo Meliconi (Head, Medical Department, IRCCS Rizzoli Hospital) for support in the study. The participation of both teams (medical and nurse personnel) is also acknowledged.

\section{Disclosure}

Giulio Marchesini reports he is on the advisory board for Gilead, Astra-Zeneca, Sanofi and Eli Lilly, outside the 
submitted work. The authors report no conflicts of interest in this work.

\section{References}

1. Pieralli F, Bazzini C, Fabbri A, et al. The classification of hospitalized patients with hyperglycemia and its implication on outcome: results from a prospective observational study in internal medicine. Intern Emerg Med. 2016;11:649-656. doi:10.1007/s11739-015-1358-6

2. Pagano E, De Rosa M, Rossi E, et al. The relative burden of diabetes complications on healthcare costs: the population-based CINECA-SID ARNO diabetes observatory. Nutr Metab Cardiovasc Dis. 2016; 26:944-950. doi:10.1016/j.numecd.2016.05.002

3. Dungan KM, Braithwaite SS, Preiser JC. Stress hyperglycaemia. Lancet. 2009;373:1798-1807. doi:10.1016/S0140-6736(09)60553-5

4. Umpierrez GE, Isaacs SD, Bazargan N, You X, Thaler LM, Kitabchi AE. Hyperglycemia: an independent marker of in-hospital mortality in patients with undiagnosed diabetes. $J$ Clin Endocrinol Metab. 2002;87:978-982. doi:10.1210/jcem.87.3.8341

5. Umpierrez GE, Kosiborod M. Inpatient dysglycemia and clinical outcomes: association or causation? J Diabetes Complications. 2014;28:427-429. doi:10.1016/j.jdiacomp.2014.03.008

6. Peralta G, Sanchez MB, Garrido JC, et al. Altered blood glucose concentration is associated with risk of death among patients with community-acquired gram-negative rod bacteremia. BMC Infect Dis. 2010;10:181. doi:10.1186/1471-2334-10-181

7. Yang CJ, Liao WI, Tang ZC, et al. Glycated hemoglobin A1c-based adjusted glycemic variables in patients with diabetes presenting with acute exacerbation of chronic obstructive pulmonary disease. Int J Chron Obstruct Pulmon Dis. 2017;12:1923-1932. doi:10.2147/ COPD.S131232

8. Capes SE, Hunt D, Malmberg K, Pathak P, Gerstein HC. Stress hyperglycemia and prognosis of stroke in nondiabetic and diabetic patients: a systematic overview. Stroke. 2001;32:2426-2432. doi:10. 1161/hs1001.096194

9. Paciaroni M, Agnelli G, Caso V, et al. Acute hyperglycemia and early hemorrhagic transformation in ischemic stroke. Cerebrovasc Dis. 2009;28:119-123. doi:10.1159/000223436

10. Harp JB, Yancopoulos GD, Gromada J. Glucagon orchestrates stress-induced hyperglycaemia. Diabetes Obes Metab. 2016;18: 648-653. doi:10.1111/dom.12668

11. Bonizzoli M, Zagli G, Lazzeri C, Degl'Innocenti S, Gensini G, Peris A. Early insulin resistance in severe trauma without head injury as outcome predictor? A prospective, monocentric pilot study. Scand J Trauma Resusc Emerg Med. 2012;20:69. doi:10.1186/1757-7241-20-69

12. Chen Y, Yang X, Meng K, et al. Stress-induced hyperglycemia after hip fracture and the increased risk of acute myocardial infarction in nondiabetic patients. Diabetes Care. 2013;36:3328-3332. doi:10.2337/dc13-0119

13. Esposito K, Nappo F, Marfella R, et al. Inflammatory cytokine concentrations are acutely increased by hyperglycemia in humans: role of oxidative stress. Circulation. 2002;106:2067-2072. doi:10.1161/01. CIR.0000034509.14906.AE
14. Losser MR, Damoisel C, Payen D. Bench-to-bedside review: glucose and stress conditions in the intensive care unit. Crit Care. 2010;14:231. doi:10.1186/cc9100

15. Bochicchio GV, Salzano L, Joshi M, Bochicchio K, Scalea TM. Admission preoperative glucose is predictive of morbidity and mortality in trauma patients who require immediate operative intervention. $\mathrm{Am}$ Surg. 2005;71:171-174.

16. Richards JE, Kauffmann RM, Obremskey WT, May AK. Stress-induced hyperglycemia as a risk factor for surgical-site infection in nondiabetic orthopedic trauma patients admitted to the intensive care unit. J Orthop Trauma. 2013;27:16-21. doi:10.1097/BOT.0b013e31825d60e5

17. Scalea TM, Bochicchio GV, Bochicchio KM, Johnson SB, Joshi M, Pyle A. Tight glycemic control in critically injured trauma patients. Ann Surg. 2007;246:605-10; discussion 10-2. doi:10.1097/SLA.0b $013 \mathrm{e} 318155 \mathrm{a} 789$

18. Roberts GW, Quinn SJ, Valentine N, et al. Relative hyperglycemia, a marker of critical ilness: introducing the stress hyperglycemia ratio. J Clin Endocrinol Metab. 2015;100:4490-4497. doi:10.1210/jc.20152660

19. Charlson ME, Pompei P, Ales KL, MacKenzie CR. A new method of classifying prognostic comorbidity in longitudinal studies: development and validation. J Chronic Dis. 1987;40:373-383. doi:10.1016/ 0021-9681(87)90171-8

20. Nathan DM, Kuenen J, Borg R, et al. Translating the A1C assay into estimated average glucose values. Diabetes Care. 2008;31:1473-1478. doi: $10.2337 / \mathrm{dc} 08-0545$

21. Chrastil J, Anderson MB, Stevens V, Anand R, Peters CL, Pelt CE. Is hemoglobin A1c or perioperative hyperglycemia predictive of periprosthetic joint infection or death following primary total joint arthroplasty? J Arthroplasty. 2015;30:1197-1202. doi:10.1016/j. arth.2015.01.040

22. Martin ET, Kaye KS, Knott C, et al. Diabetes and risk of surgical site infection: a systematic review and meta-analysis. Infect Control Hosp Epidemiol. 2016;37:88-99. doi:10.1017/ice.2015.249

23. Mraovic B, Suh D, Jacovides C, Parvizi J. Perioperative hyperglycemia and postoperative infection after lower limb arthroplasty. J Diabetes Sci Technol. 2011;5:412-418. doi:10.1177/1932296 81100500231

24. Agos F, Shoda C, Bransford D. Part II: managing perioperative hyperglycemia in total hip and knee replacement surgeries. Nurs Clin North Am. 2014;49:299-308. doi:10.1016/j.cnur.2014.05.004

25. Bock M, Johansson T, Fritsch G, et al. The impact of preoperative testing for blood glucose concentration and haemoglobin A1c on mortality, changes in management and complications in noncardiac elective surgery: a systematic review. Eur $J$ Anaesthesiol. 2015;32:152-159. doi:10.1097/EJA.0000000000000117

26. Dhatariya K, Levy N, Kilvert A, et al. NHS diabetes guideline for the perioperative management of the adult patient with diabetes. Diabet Med. 2012;29:420-433. doi:10.1111/j.1464-5491.2012.03582.x

27. Akiboye F, Rayman G. Management of hyperglycemia and diabetes in orthopedic surgery. Curr Diab Rep. 2017;17:13. doi:10.1007/ s11892-017-0839-6

28. Levy N, Dhatariya K. Pre-operative optimisation of the surgical patient with diagnosed and undiagnosed diabetes: a practical review. Anaesthesia. 2019;74(Suppl 1):58-66. doi:10.1111/anae.14510 


\section{Publish your work in this journal}

Diabetes, Metabolic Syndrome and Obesity: Targets and Therapy is an international, peer-reviewed open-access journal committed to the rapid publication of the latest laboratory and clinical findings in the fields of diabetes, metabolic syndrome and obesity research. Original research, review, case reports, hypothesis formation, expert opinion and commentaries are all considered for publication. The manuscript management system is completely online and includes a very quick and fair peer-review system, which is all easy to use. Visit http://www.dovepress.com/testimonials.php to read real quotes from published authors.

Submit your manuscript here: https://www.dovepress.com/diabetes-metabolic-syndrome-and-obesity-targets-and-therapy-journal 